\title{
Interval Size and Affect: An Ethnomusicological Perspective
}

\author{
SARHA MOORE \\ The University of Sheffield
}

\begin{abstract}
This commentary addresses Huron and Davis's question of whether "The Harmonic Minor Provides an Optimum Way of Reducing Average Melodic Interval Size, Consistent with Sad Affect Cues" within any non-Western musical cultures. The harmonic minor scale and other semitone-heavy scales, such as Bhairav raga and Hicaz makam, are featured widely in the musical cultures of North India and the Middle East. Do melodies from these genres also have a preponderance of semitone intervals and low incidence of the augmented second interval, as in Huron and Davis's sample? Does the presence of more semitone intervals in a melody affect its emotional connotations in different cultural settings? Are all semitone intervals equal in their effect? My own ethnographic research within these cultures reveals comparable connotations in melodies that linger on semitone intervals, centered on concepts of tension and metaphors of falling. However, across different musical cultures there may also be neutral or lively interpretations of these same pitch sets, dependent on context, manner of performance, and tradition. Small pitch movement may also be associated with social functions such as prayer or lullabies, and may not be described as "sad." "Sad," moreover may not connote the same affect cross-culturally.
\end{abstract}

Submitted 2012 Nov 24; accepted 12 December 2012.

KEYWORDS: sad, harmonic minor, Phrygian, Hicaz, Bhairav, Yishtabach

HURON and Davis's article states that major scale melodies, on having their third and sixth degrees flattened, contain smaller intervals on average, and that if small pitch movement connotes "sadness," then altering a standard major melody to the harmonic minor is "among the very best pitch-related transformations that can be done to modify a major-mode melody in order to render a sad affect" (p. 105, 114). I look forward to the testing of this hypothesis, playing these pieces to "major-scale enculturated" listeners to find out their opinions.

Although Huron and Davis's study is focused on the Western-enculturated listener, I am addressing their interest in extending this study to cross-cultural situations (p. 103, 114). Empirical Musicology Review has an established history of interdisciplinary discussion between music psychology and ethnomusicology, as in vol. 2, no. 4 where Martin Clayton and John Baily discuss how the beginnings of Ethnomusicology's predecessor, Comparative Musicology, were in the Institute of Psychology in Berlin. Clayton argues that both disciplines are "inherently interdisciplinary" (Clayton, 2009, p. 75); while Baily states that he has used what he knows of psychology to "understand more about processes of music cognition" within his work in Afghanistan-also suggesting that music psychologists might "change the parameters slightly" to include cross-cultural samples (Baily, 2009, p. 86).

Cognitive psychology studies are generally conducted within a Western setting, and Huron and Davis are at pains to point out that in some cultures there are no particularly "sad" associations to the harmonic minor scale (p. 104). People who have not been "Western-enculturated" in their listening habits as children may have very different perceptions. My research and ethnographic interviews, including within North Indian classical and music of the Middle East, have turned up relevant comments concerning "sad" connotations of scales. Huron and Davis's results hinge on the "relative absence of melodic "traffic" between pitch degrees 6 and 7, the augmented second interval within a harmonic minor scale, in the tunes selected for study, and the extra semitone interval between pitch degrees 5 and 6 . I will focus on the semitone interval: Does the general presence of more semitone intervals make a piece sound "sadder"? Are all semitone intervals equal? I also consider what we mean by the word "sad." 


\section{THE CONNOTATIONS OF THE SEMITONE INTERVAL}

The harmonic minor scale has three semitone intervals, compared to the two in a major scale. Beyond speech prosody, what extra-musical connotations are there connecting the semitone and "sadness"? The association of semitones with negative emotions is strong in the West: "large pitch variation may be associated with happiness, pleasantness, activity, or surprise; small pitch variation with disgust, anger, fear or boredom" (Gabrielsson \& Lindström, 2010, pp. 240-1). Since Pythagoras and Plato there have been connotative links between the semitone and dysfunction, incompleteness, and effeminacy. The harmonic series identified unequal pitch steps and the "problem" of the semitone (Leach, 2006, p. 1). Leach reports that in the Medieval era the term "semi" was regarded by authors such as Guido d'Arezzo as meaning an "incomplete" tone. Connections were even made between the semitone "leading note" attraction to the tonic and the leading of the "simple and masculine" to the "effeminate and violent." Music "rich in intervals smaller than a tone" was thus deemed to be of a "morally dubious nature" (Leach, 2006, pp. 2, 5), in a move most would now surely be wary of making.

\section{Metaphors}

Many binary metaphors that relate to large and small intervals are based on the body, with "Joy is Wide; Sadness is Narrow" perhaps being based on the expansive feeling of a smile (Lakoff \& Johnson, 1980, p. 18). Cross-modal mapping takes this to the notion of expansive intervals being best for joyful music, narrow for sadness. The energy required to perform large intervals is also sometimes connoted as "Power is Wide; Weakness is Narrow" (Kivy, 1989, pp. 39, 41, 55). Empirical studies on Western emotional responses to melodic intervals include Maher and Berlyne's 1982 study using 12 binary rating scales, which finds that small intervals are considered weaker, simpler, more indefinite, and melancholic than larger intervals. In the specific case of the semitone, it is considered the most melancholic, and more tense and more beautiful than most other intervals (Maher \& Berlyne, 1982, p. 16). The, perhaps, surprising introduction here of the concept of beauty takes us away from binary thinking and towards nuanced connotations, including those of the semitone interval that I have encountered within non-Western cultures.

\section{A VIEW FROM ETHNOMUSICOLOGY}

In an interview, Indian Sitar player Baluji Shrivastav (personal communication) uses the generic term "expressive" to give the idea of a semitone interval's capacity to carry meaning in music. He describes how: "the closer notes are very expressive. Anything that is closer, you can feel more expression, it's very physical. If you play different notes, the closer you get, the vibrato gets faster and stronger." Shrivastav describes the semitone nearest to the tonic as the most expressive, the most "powerful." This conflicts with the (above) interpretation of small intervals as weaker.

The "power" of this "leading note" may, perhaps, be more "expressive" within a tonal system than other semitone intervals. Bharucha's model of melodic anchoring describes the pull, especially of the semitone, towards stable pitches in the scale, as like an arrow "yearning" towards the stable tone, a "psychological force pulling a musical event up or down," with the notes that are a semitone away from the tonic having the greatest "yearning" effect (Bharucha, 1996, pp. 383, 398). These concepts of "attraction" may also be emotionally neutral, a structural feature, as when Chew addresses the "attractive force" of the major 7th leading note towards the tonic, noting its centrality to Common Practice tonality (Chew, 1983, p. $35)$.

\section{The Harmonic Minor may not be "Sad"}

In some musical cultures the harmonic minor is a fundamental scale. Yossi Sa'aron, an Israeli guitarist (personal communication), says that, for him, the harmonic minor is the "most natural thing; far from dissonant; more normal and natural than Ionian or Dorian." When composing, he starts with the harmonic minor, with its "half-tone, magical" qualities, and "everything else is variations of it," introducing what he calls "flat scales" such as the Aeolian "if I need something for the mood, for the sadness of a piece or phrase." Seemingly, then, Huron and Davis's conclusions are refuted by these remarks. However, there may be other factors at play, particularly the issues of cultural familiarity.

The musical culture that surrounds you in life clearly determines what you regard as aurally "normal." It has come up in various studies that on hearing notes that are different to "normal" we have particular and specific reactions. For instance, a study by Huron, Yim, and Chordia's found an association 
between sadness and "lower than normal pitch," where the realization of melodic expectation contributed to a feeling of pleasure (Huron, Yim, \& Chordia, 2010, pp. 63-6). The most well-trodden melodic path in Huron and Davis's probability study of different scale-tone successions, in Germanic major mode folk melodies, is the 4-3-2-1 tetrachord (see Fig.2 p. 107). If the 2nd and 3rd degree were flattened to give the tetrachord $4-b 3-b 2-1$, the average melodic size may stay roughly the same. But does the latter sound sadder to those enculturated to the major scale? One might expect that the harmonic minor scale may not evoke as strong an emotional reaction as very unfamiliar modes such as the Locrian or Phrygian modes. Research may shed light on whether this factor would outweigh general effects of interval size.

The sound of weeping, heard as a falling semitone, became an iconic musical symbol of grief, pain and loss in Western Classical music. Semiotician Raymond Monelle writes that "the moan of the dissonant falling second expresses perfectly the idea of lament" (Monelle, 2000, p. 72). Shrivastav (personal communication) agrees that for him, too, the falling semitone is sad, but continued that the same interval rising is not sad: "This is quite a happy aspect, going back down is the sad aspect."

In Jewish Liturgical music there is a mode called the Yishtabach mode that principally uses the Aeolian mode, except at cadences where the second degree is flattened. Professor Alexander Knapp (personal communication) told me that the flattening of the second here is: "A colouristic effect, making a more effective cadence, somehow reflecting the prose of the liturgy of devotion."

This "lower than normal" note, then, is used for adding expressive meaning, such as of "sadness" or "devotion." Familiarity on the one hand, and "falling" from "normal" on the other, may influence interpretations as much as actual interval size.

\section{Musical Motifs and Ornamentation}

Within North Indian classical music the notes equivalent to the Western major scale are called shuddha (natural) notes, other pitches being called "altered" (Sobhana, 1989, pp. 132). Singer Subroto Roy (personal communication) gives a different perspective to flattened pitches, concerning their use in music played at dawn and dusk: "flattened notes help you to get into the active mode of life, or make you sleep well, they bridge between the unconscious and conscious minds, or between different levels of consciousness." Roy here opens up a new world of interpretations that are affected by extra-musical association and how notes are treated: "You can't say that flat notes are sad, it all depends on the context, the relationship with other notes, and your traditional understanding." Roy thus highlights the radical differences between Western melodic concepts and Indian raga, where ragas are defined by the uniqueness of their notes.

This, to a greater or lesser extent, applies to the classical musics of Turkish makam and Arabic maqam that also have strong connections between "scale," musical motifs, and context. It would be challenging to attempt Huron's present study within any of these genres, as altering pitch degrees destroys the concept of a raga or makam/maqam, rendering it simply "wrong," with the musical motifs of one raga/ makam/maqam and the notes of another. It might be possible to make the interval-size calculation but it would be meaningless in terms of the actual music. The equivalent change within Western music results in a piece that may feel "wrong" in terms of a particular piece, yet changing the tonality into a harmonic minor would, I suggest, still be perfectly acceptable as a piece within the style/genre.

\section{Empirical Evidence within South Indian Music}

An empirical study of Carnatic Classical Music addresses a similar issue to Huron and Davis's: Bowling Sundarajan, Han, and Purves (2012) compare the distribution of melodic intervals between melodies in ragas associated with the "positive/excited" rasa (emotion) Hasya, and those connected with the "negative/ subdued" rasa Karuna. I would point out here that the "subdued" aspect of these ragas is not tied to the "negative" in any "bad" sense: the rasa Karuna is "compassion" which may have associations of pathos, love, sympathy, and tenderness. The two "positive/excited" ragas are based on notes equivalent to the major scale, and the three "negative/subdued" ragas all have b2 and b6, together (in two cases) with other "altered" notes.

Bowling et al. (2012) find the principal difference to be the proportion of intervals smaller or larger than a major second. Their results are that there are significantly more semitone intervals in the "negative/subdued" raga melodies, making a correlation between these results and scales with flattened pitch degrees. This study highlights the prevalence of the $b 6$ in the "negative/subdued" raga melodies, rather than the $b 3$ in their comparative American study, concluding that "the use of a particular tonic interval(s) is not critical for the expression of emotion" (Bowling et al., 2012). So, put alongside Huron and 
Davis's study, this is the primary difference: the flattening of notes from a "major scale" makes for smaller intervals generally, but Bowling et al. find no particular flattened notes to hold special significance.

\section{CONTRADICTIONS AND SUBTLETIES OF CONNOTATION}

Within North Indian Classical music the ragas Bhairav, Bhairavi and Todi correspond to the three "negative/subdued" ragas studied above. They also are associated with rasa Karuna, each with particular characters: Bhairav is considered a devotional raga, Bhairavi a light, loving raga (Bor, 1996, pp. 32, 34), and Todi has associations of abandonment (Goswami, 1995, p. 42). None would be regarded as simply "negative." For instance, raga Bhairav with its four semitone intervals, is described by Roy (personal communication) as, for him, evoking a "delight, a spiritual bliss, as when meditating." Sufi singer Rafaqat Ali Khan (personal communication) described the presence of a b2 as "sad" and said: "Sad in our Sufi religion doesn't mean: 'Oh, I've lost my purse,' it means that I'm closer to God, it is a beautiful and lovely sensation." Shrivastav (personal communication) agreed that "sad" was how he, too, understood a b2: "sad, and relaxed.... Sad can be romantic, when you long for someone, a longing mood." So the question arises of what we mean when we use the word "sad."

Small melodic intervals, low frequency, slow tempo, smooth and with quiet dynamics are the parameters identified by Huron and Davis that convey "sadness" (p. 104). However, Clayton finds the terms "calm" and "sad" both to be reactions to raga Shri, another raga with four semitone intervals, and surmises that "both ['calm' and 'sad'] would be associated with slow introverted movements" within that raga (Clayton, 2005, p. 371). And Johnstone and Scherer describe how "joy" may be a quiet emotion expressed with small speech movements (Johnstone \& Scherer, 2000, p. 229). Huron and Davis acknowledge "sleepy" as another interpretation of these parameters (p. 103), and Turkish violin player Cahit Baylav (personal communication) told me that the makam Hicaz (comparable to a mode of the harmonic minor) was used for a large proportion of lullabies. He further gave his opinion that makam Hicaz was very popular with the adult Turkish population for this nostalgic connection. These parameters, then, including small melodic intervals, might combine to express more than "sadness" in different cultures: in a prayer, a lullaby, or a joyful or tender musical expression, not as exceptions but as widespread phenomena.

Huron and Davis also write of how small melodic movement may be interpreted very differently when treated in a different way (p. 104). For instance, the North Indian raga Basant comes from the same pitch set as raga Shri, yet Shri is always serious, while Basant is played faster and lightly, for the joyous, playful Spring holi festival. Another instance is how the scales of Jewish liturgical song are transformed in Klezmer, Eastern European dance music. The "sad," devotional song "Ahava Raba," using the same pitch set as Hicaz makam, becomes a lively, joyful dance tune, often with accentuated semitone intervals.

\section{"Melancholic Airs of the Orient"}

Interestingly, the interval whose relative absence in Huron and Davis's study has produced a low average interval size in the harmonic minor, the augmented second between the 6th and 7th degrees (p. 113), itself receives connotations of "sad" affect. For instance, in the Bosnian musical genre Sevda, that takes its name from a word related to melancholia. Milošević associates its "melancholia" with the augmented second interval (Milošević, quoted in Pennanen, 2010, p. 78). Pennanen writes, of the augmented second, that "Muslim Slavs adopted the interval because it reflects love's yearnings and expressions of Oriental melancholy" (Pennanen, 2010, p. 80). However, Bosnian accordionist Merima Ključo (personal communication) attributes the "loving" affect to the semitone flattened second: "it's so interesting to see people react when you just change the second note.... all of a sudden your body moves, your ear, everything just turns to the different direction. It is like looking to the wonderful baby and giving a kiss." Augmented second or "lower" than normal flattened second, melancholia or tenderness, varying interpretations abound. Ironically, considering Huron and Davis's hypothesis, melodic "traffic" between the 6 th and 7th degrees may actually add to "sad" associations for the harmonic minor. 


\section{CONCLUSIONS}

The variance of structural melodic concepts in classical musics from North India and Turkey may preclude a repetition of Huron and Davis's study there, though related studies may produce comparable results. Melodies in the harmonic minor scale, and other scale types that contain many semitones, receive wide and varied associations in different musical cultures. The semitone is associated with the "sad" and the "beautiful," it can be "joyful," "tense," or "neutral," and some semitone intervals are considered to be of greater affect than others. Familiarity, metaphorical differences, ideologies and cultural meanings introduce other perspectives in the study of interval size within and across cultures, that offer an opportunity to embed cultural diversity in cognitive musical discourse.

\section{INTERVIEWS}

Interviews conducted by Sarha Moore between 2008 and 2012:

Cahit Baylav, Turkish violin player based in London

Rafaqat Ali Khan, Pakistani Sufi singer based in Lahore

Merima Ključo, Bosnian accordionist based in Amsterdam

Alexander Knapp, pianist, Professor Emeritus of Jewish Music based in London

Subroto Roy, Indian singer based in Pune

Yossi Sa'aron, Israeli guitarist based in Tel Aviv

Baluji Shrivastav, Indian sitar player based in London

\section{REFERENCES}

Baily, J. (2009). Crossing the boundary: From experimental psychology to ethnomusicology. Empirical Musicology Review, Vol. 4, No. 2, pp. 82-88.

Bharucha, J. (1996). Melodic anchoring. Music Perception: An Interdisciplinary Journal, Vol. 13, No. 3., pp. 383-400.

Bor, J. (1999). The Raga Guide: A Survey of 74 Hindustani Ragas. Rotterdam: Nimbus Records.

Bowling, D. L., Sundarajan, J., \& Han, S., \& Purves, D. (2012). Expression of emotion in Eastern and Western music mirrors vocalization. PLOS ONE.

http://www.plosone.org/article/info:doi/10.1371/journal.pone.0031942

Chew, G. (1983). The spice of music: Towards a theory of the leading note. Music Analysis, Vol. 2., No. 1, pp. 35-53.

Clayton, M. (2005). Communication in Indian raga performance. In: D. Miell, D. Hargreaves, \& R. MacDonald (Eds.), Musical Communication. Oxford: Oxford University Press, pp. 361-381.

Clayton M. (2009). Crossing boundaries and bridging gaps: Thoughts on relationships between ethnomusicology and music psychology. Empirical Musicology Review, Vol. 4, No. 2, pp. 75-77.

Gabrielsson, A., \& Lindström E. (2010). The role of structure in the musical expression of emotions. In: P.N. Juslin, \& J.A. Sloboda (Eds.), Handbook of Music and Emotion: Theory, Research, Applications. Oxford: Oxford University Press, pp. 367-400.

Goswami, R. (1995). Meaning in Music. Shimla: Indian Institute of Advanced Study.

Huron, D., Yim, G., \& Chordia, P. (2010). The effect of pitch exposure on sadness judgments: An association between sadness and lower-than-normal pitch. In: S.M. Demorest, S.J. Morrison, \& P.S. Campbell (Eds.), Proceedings of the 11th International Conference on Music Perception and Cognition, pp. 63-66. 
Johnstone, T., \& Scherer, K.R. (2000). Vocal communication of emotion. In: M. Lewis, Haviland-Jones (Eds.), Handbook of Emotions. New York: Guilford Press, pp. 220-235.

Kivy, P. (1989). Sound Sentiment. Philadelphia: Temple University Press.

Lakoff, G., \& Johnson, M. (1980). Metaphors We Live By. Chicago: University of Chicago Press.

Leach, E.E. (2006). Gendering the semitone. Music Theory Spectrum, Vol. 28, No. 1, pp. 1-22.

Maher, T., \& Berlyne, D.E. (1982). Verbal and exploratory responses to melodic musical intervals. Psychology of Music, Vol. 10, pp. 11-27.

Monelle, R. (2000). The Sense of Music: Semiotic Essays. Princeton: Princeton University Press.

Pennanen, R.P. (2010). Melancholic airs of the Orient-Bosnian Sevdalinka music as an Orientalist and national symbol. In: R.P. Pennanen (Ed.), Music and Emotions. Helsinki: University of Helsinki, pp. 76-90. http://hdl.handle.net/10138/25832

Sobhana, N. (1989). Bhatkhande's Contribution to Music: A Historical Perspective. Delhi: Popular Prakashan. 